\title{
GESTIÓN DE RECURSOS HUMANOS: ENFOQUE SISTÉMICO EN UNA PERSPECTIVA GLOBAL
}

\author{
José LUIS Perea RIVERA*
}

\section{RESÚMEN}

La temática tiene como propósito abordar los principales procesos de la gestión de los recursos humanos en las organizaciones de producción de bienes y servicios (empresa, industria, institución) sea del sector público o privado, que en su dinámica operacional está presente el componente psicológico. También trata su interrelación que, bajo un enfoque sistémico y en la perspectiva global hacia la modernidad del siglo XXI dentro de la concertación y el cambio organizacional, sirva de soporte en la sistematización de la gestión; asimismo, que propicie la consistencia entre la planificación, la evaluación para fines del mejoramiento contínuo, acreditación o certificación de calidad de sus procesos, y de la pertinencia con las demandas de la realidad social, económica y política del país.

Palabras claves: Cambio organizacional, captación, habilitación, calificación, potenciación.

\begin{abstract}
The thematic one must like intention to approach the main processes of the management of the human resources in the organizations of production of goods and services (company, industry, institution) is of the public or deprived sector, that in its operational dynamics is present the psychological component. Also it treats his interrelation that under a sistémico approach and in the global perspective towards the modernity of century XXI within the agreement and the organizacional change, serves as support in the systematization of the management; which it causes the consistency between the planning, the evaluation for aims of the continuous improvement, accreditation or certification of quality of his processes, and the pertinencia with the demands of the social reality, economic and also political of the country.
\end{abstract}

Keywords: Organizacional change, gathering, habilitation, qualification, potentiality.

* Docente de la Facultad de Psicología de la UNMSM. E-mail: jperear@unmsm.edu.pe 


\section{INTRODUCCIÓN}

El contenido del presente trabajo tiene un marco referencial multidisciplinario, del cual toma como base las funciones administrativas determinadas por Koontz y Weihrich [1], que corresponde a la Integración de Personal, cuyo objetivo es «garantizar que las funciones organizacionales sean desempeñadas por personal calificado idóneo y dispuesto a ejercerlas», y responde al principio de «cuanto más claro sea la definición de las funciones organizacionales y sus requerimientos humanos, y cuanto mejores sean las técnicas que se empleen en la selección, evaluación y capacitación de los administradores, tanto mayor será la calidad administrativa de una empresa.»

Asimismo, esta función administrativa la definen como el «cubrir y mantener cubiertos los puestos de la estructura organizacional.», para lo cual se «...requiere la identificación de las necesidades de recursos humanos y de la ocupación en la estructura organizacional, y su conservación en este estado, con personas competentes.», y se logra «cuando desarrollamos una serie de acciones para identificar los requerimientos de la fuerza de trabajo, realizar un inventario del personal disponible y reclutar, seleccionar, contratar, remunerar, evaluar, planear las carreras, capacitar o desarrollar, y ascender en alguna otra forma tanto a los candidatos como a los titulares de los puestos, para que puedan cumplir con sus tareas de un modo eficaz y eficiente.». Otro término que se usa con frecuencia para la función administrativa de integración de personal es la «administración de los recursos humanos».

En esta perspectiva, encontramos que Bohlander, Snell y Sherman [2] enfatizan que «El papel de los gerentes de RH ya no está limitado a funciones de servicio como el reclutamiento y la selección. Hoy día asumen un papel activo en la planeación estratégica y la toma de decisiones en sus organizaciones. "...«Las organizaciones en el mundo competitivo actual están descubriendo que lo que hace toda la diferencia es cómo se combinan los temas particulares de los RH. En general, los gerentes no se centran de manera aislada en aspectos de los RH como contratación, capacitación y compensaciones. Cada una de las tareas de recursos humanos se combina en un sistema global para mejorar la participación y la productividad de los trabajadores.»

\section{PRESENTACIÓN}

Al tratar en este caso particular, los campos de aplicación de la Psicología en el ámbito organizacional, nos estaremos refiriendo a la línea del trabajo psicológico en Apoyo a la Gestión de la organización (funcional u operativo), resultante de un planeamiento estratégico para la dinámica humana y de la organización [3], y específicamente a la Administración de Recursos Humanos dentro de un enfoque sistémico.

En las organizaciones (empresas-industrias e instituciones) la gestión de los recursos humanos, generalmente, ejecuta sus procesos de trabajo bajo lineamientos o criterios de naturaleza administrativa, no obstante, se puede identificar dos líneas de trabajo bien diferenciadas, una de ellas con propósito y naturaleza netamente administrativas, y la otra línea de trabajo a procesos/programas cuya ejecución requiere permanentemente a una relación social laboral en su ejecución, es decir, en la dinámica de su gestión está presente el componente psicológico. 


\section{Delimitando Líneas o Procesos de Trabajo}

Para una mejor planificación, ejecución y control de la administración/gestión de recursos humanos, se deberá tomar en cuenta para obtener resultados exitosos, que todos los procesos no tienen o no responden a una misma naturaleza o tipo de dinámica, por lo que se recomienda tratar de realizar la delimitación de estas líneas o procesos de trabajo, para tal efecto se presenta el alcance siguiente:

1. Procesos de trabajo de naturaleza administrativa, cuya ejecución responde a la aplicación de directivas, reglamentos o normas, que para el logro de sus objetivos o metas, así como para enriquecer la información de los trabajadores, su eficiencia o la dinámica de su gestión dependerá básicamente de las relaciones que devienen del avance de la tecnología, y no necesariamente, como producto de las relaciones social laborales-interacción humana.

Entre los procesos de naturaleza administrativa tenemos al Control de Personal, que desde el registro en cuadernos o listas de asistencia, pasó hacia la impresión de tarjetas de asistencia por un marcador de reloj, después a la lectura de código de barras mediante el fotocheck, hasta el control del registro mediante la voz, a la lectura de la huella digital, de la mano o el ojo, por ahora. Otros procesos son el Procesamiento de Planillas, presupuestos, cuentas corrientes del personal; el Procesamiento de beneficios laborales, escalafón, movimiento de personal, etc.

Estos procesos o líneas de trabajo debido a las bondades que proporcionan los equipos de procesamiento más sofisticados cada día, limitan o anulan cada vez más la presencia o la relación interpersonal, igual efecto producen el uso exagerado de formatos preimpresos y las diversas formas de presentación y posterior recepción de documentos internet, que también reducen casi al máximo la relación interpersonal.

2. Procesos de trabajo con componente psicológico, requieren necesariamente de la permanente relación social laboral, los cuales deberán estar a cargo o monitoreados por personal profesional para que brinden asistencia técnica, orientación y asesoramiento especializado, que permitirán garantizar el logro exitoso de sus metas y de los objetivos de la institución, como resultado de la internalización de los propósitos que tiene la organización, de la visión compartida y del compromiso de la misión por todos los trabjadores, sea cual fuere su nivel de responsabilidad o estatus.

\section{MODELO DE GESTIÓN}

«... el cambio organizacional se describe como «cambio profundo» que combina modificaciones internas de los valores de la gente, sus aspiraciones y conductas, con «variaciones externas» en procesos, estrategias, prácticas y sistemas.

En el cambio profundo hay aprendizaje. No basta con cambiar estrategias, estructuras y sistemas, también tienen que cambiar las maneras de pensar...»

PETER SENGE, La Danza del Cambio.[4] 
En la Gestión de Recursos Humanos para los procesos de trabajo con componente psicológico deberán ejecutarse bajo un enfoque sistémico, que propiciando la sinergia entre todas las áreas de trabajo, beneficiará a todos a los usuarios, internos-externos y facilitará al crecimiento y desarrollo saludable de la organización como un todo.

En esta perspectiva, planteamos una propuesta de sistematización de las líneas de trabajo con componente psicológico, [5] cuya secuencialidad de ejecución a nivel de procesos no es rígida, puede variar, sea por la política de la organización y/o por la característica de temporalidad de la ejecución de sus programas, que son cíclicos-eventuales como contínuospermanentes.

\section{PROCESOS}

1. Captación:

2. Habilitación:

3. Calificación:

4. Potenciación:

\section{PROGRAMAS}

Reclutamiento, Selección e Inducción.

Formación y Capacitación.

Evaluación de Personal

Desarrollo de Personal.

Estos procesos aparecen en el Cuadro 1 «Gestión Sistémica de los Recursos Humano: Componente Psicológico», con la indicación de sus programas y el tipo de ejecución para su objetivo-usuario.

\section{EL PROCESO DE CAPTACIÓN DE RECURSOS HUMANOS}

Es uno de los procesos más importantes en la gestión de recursos humanos, debido a la toma de decisiones que se tienen que optar en situaciones donde confluyen intereses comunes entre el candidato y la organización. Ambos, la organización y el postulante realizan esfuerzos paralelos para exponer su mejor presentación, por un lado, la organización trata de obtener los mejores recursos humanos del mercado laboral y, los postulantes con su interés de querer el puesto de trabajo, sea porque él considera importante a la organización o para acceder a una fuente que le permita satisfacer otras expectativas individuales.

Asimismo, el Proceso de Captación de recursos humanos es de vital importancia para la organización, ya que del éxito de la ejecución de sus programas dependerá la atención satisfactoria de las necesidades de nuevo personal que responda a los requerimientos técnicos de sus unidades productivas o administrativas.

En resumen, el Proceso de Captación de recursos humanos tiene como propósito de armonizar la satisfacción de las necesidades de los actores del mercado laboral: la organización y la persona; y se ejecuta a través de los programas de Reclutamiento, Selección e Inducción de Personal. 
Cuadro 1. Gestión sistémica de los recursos humanos. Componente Psicológico

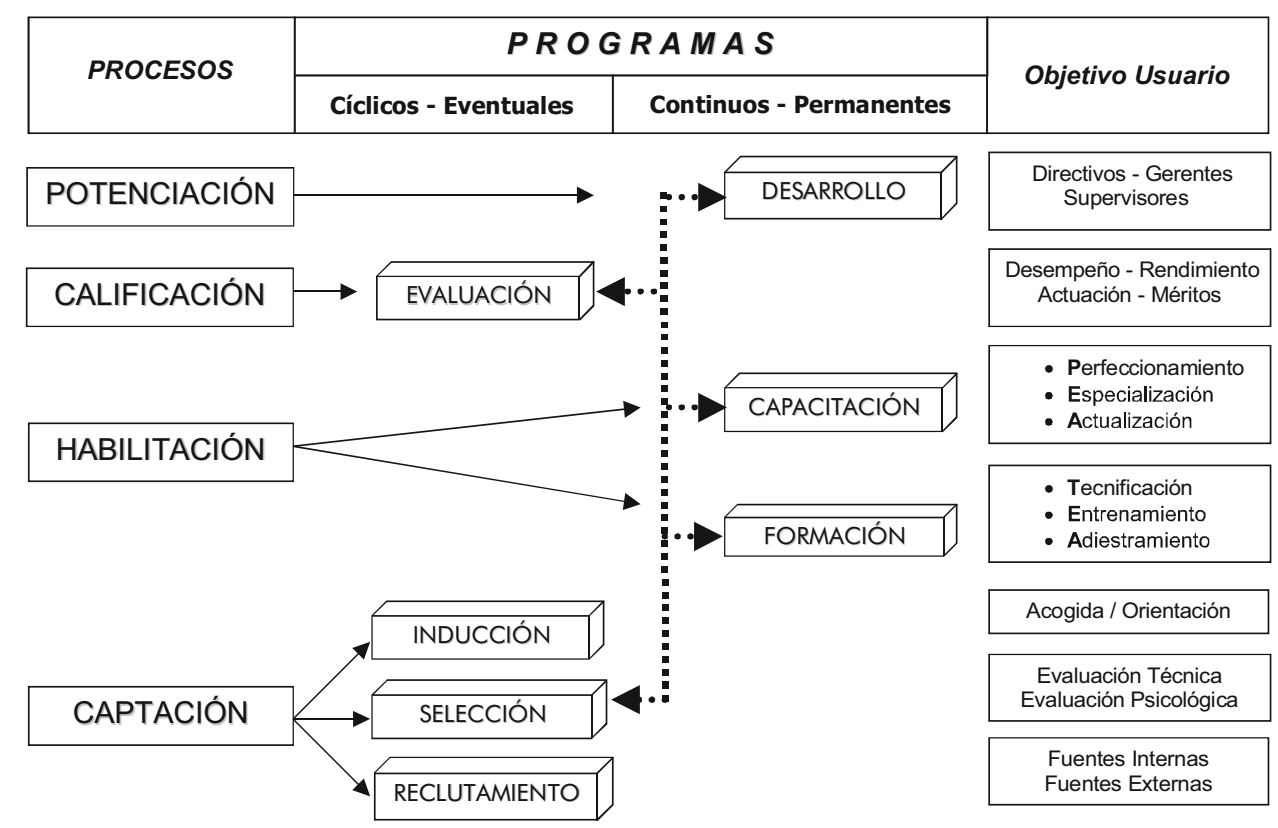

Elaboración: José Luis Perea Rivera

\section{Programa de Reclutamiento de personal}

El Reclutamiento de personal es uno de los programas más importantes en el Proceso de Captación de recursos humanos, que requiere de un exigente planeamiento de sus actividades, a fin de que la ejecución de sus eventos logre una eficaz cobertura de sus vacantes.

Previamente a su ejecución se deberá tener identificados los puestos organizacionales, el conocer las características individuales para cada puesto de trabajo, y en el mejor de los casos, conocer el plan administrativo de requerimientos de recursos humanos para saber las causas que originan las vacantes a ofertar, y dar el tratamiento más adecuado para cada caso, sea para la promoción, reubicación, renuncia o jubilación del trabajador, o por la ampliación o el desarrollo de la organización.

A continuación se presenta una propuesta de sistematización de trabajo del Programa de Reclutamiento, que aparece en el Cuadro 2, cuya ejecución puede darse a través de tres fases: las dos primeras son preparatorias y la tercera fase es propiamente la ejecución del programa. 


\section{PROGRAMA DE RECLUTAMIENTO}

\section{Cuadro 2}

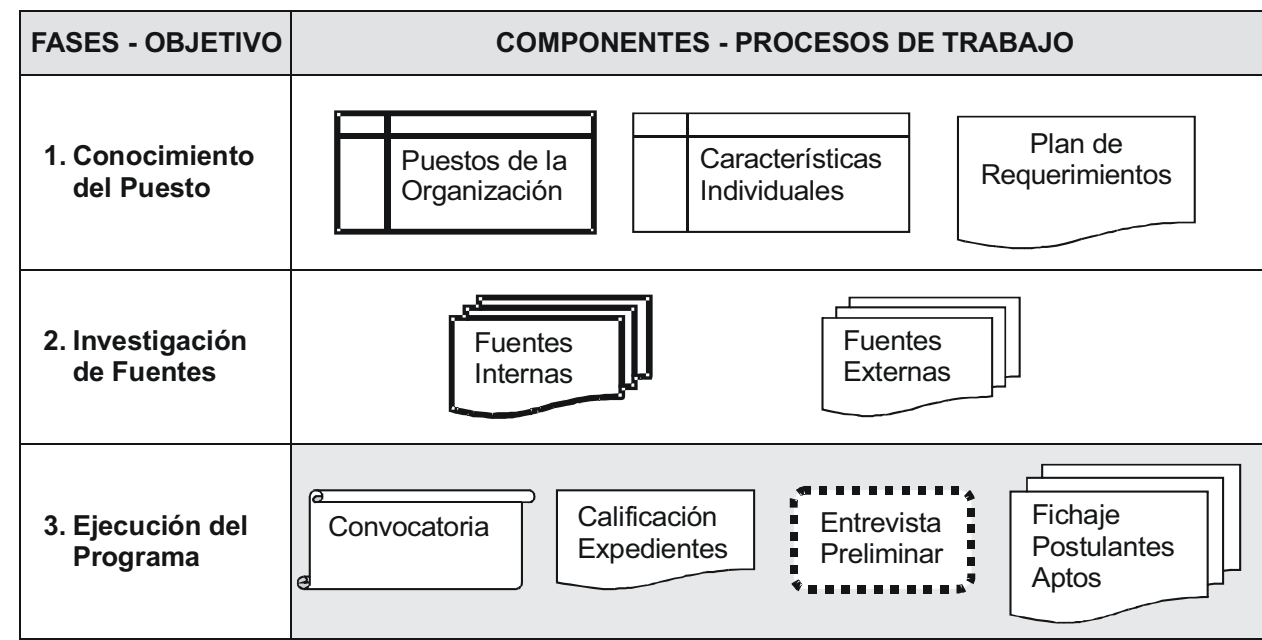

La fase 1 tiene como componentes-objetivos, el conocer los puestos organizacionales en general, las características individuales de los puestos de trabajo y el plan de requerimientos de recursos humanos; todo esto viene a ser el soporte técnico del programa de Reclutamiento.

La fase 2 tiene como componentes-objetivos, la investigación o evaluación de las posibles fuentes de captación de recursos humanos -internas y externas- . Es recomendable comenzar por la fuente interna, en razón que se producen efectos positivos (clima social laboral saludable, satisfacción personal) los que benefician tanto a la organización como al trabajador.

De no existir o satisfacer la fuente interna de proporcionar candidatos, se continuará la búsqueda en las fuentes externas, recomendándose que se comience con instituciones de estudios calificadas (institutos superiores, universidades) solicitando información sobre sus egresados sobresalientes, y de no existir o satisfacer de candidatos en esta opción, es decir, agotadas todas estas posibilidades, recién se procedería al reclutamiento abierto o público en el mercado laboral.

La fase 3 es en sí la ejecución del Programa de Reclutamiento, cuyos procesos de trabajo son: 1. la Convocatoria, 2. Calificación de expedientes, 3. Entrevista Preliminar, 4. Fichaje de postulantes aptos.

1. Convocatoria. Puede realizarse por cualquier medio de comunicación: radio, televisión y preferentemente en diarios de mayor circulación de la localidad. El mensaje deberá contener como mínimo la información sobre: Identificación de la organización, Identificación del puesto de trabajo, Funciones principales, Exigencias técnicas del puesto de trabajo, Cualidades personales, incluyendo las habilidades, destrezas, etc., Ofertas de la organización: sueldo, comisiones, beneficios, etc., el lugar y fecha de presentación del currículum. 
2. Calificación de expedientes. Este proceso de trabajo no tiene características selectivas de los postulantes, su objetivo es verificar y/o comparar la información del Currículum con la información contenida en la Convocatoria, y deberá efectuarse bajo estricto control, es decir, sólo quedarán aceptados los expedientes que respondan directamente a las exigencias técnicas y presonales del puesto de trabajo, tratándose de lograr bajo este criterio una depuración de un $50 \%$ del total de los expedientes presentados.

3. Entrevista preliminar. Este proceso de trabajo es importante porque genera el primer contacto social del postulante con la organización, su ejecución permite consistenciar en forma directa la información presentada en el currículo, conocer presencialmente y poder detectar limitantes en el postulante. Se recomienda una depuración del $50 \%$ del total de expedientes aceptados en la calificación de expedientes.

4. Fichaje de postulantes aptos. Este proceso de trabajo es simple y trata de clasificar y ordenar los expedientes de los postulantes que pasaron la depuración de los procesos 2 y 3 .

Con esta actividad se concluye la ejecución del Programa de Reclutamiento, procediéndose a la elaboración de la relación de los «postulantes aptos» que pasan al Programa de Selección de personal.

En nuestro propósito de coadyuvar al desarrollo de las organizaciones, se pone a vuestra disposición una fórmula cuya aplicación permitirá: obtener una muestra que responda en forma efectiva a las exigencias del puesto, a lograr que sólo participen los postulantes potenciales resultantes de una estricta depuración y consistencia de requerimientos técnicos e individuales en el Programa de Selección, y que cuantitativamente, se trabaje con el $25 \%$ del universo de postulantes convocados, logrando que la organización tenga menor gasto en trabajo, economía y tiempo.

En esta consideración se aplicará la fórmula:

$\mathbf{R e c} \mathbf{P P}=(\boldsymbol{\Sigma}$ Conv - 50\% Cexp ) - 50\% Eprelim

Donde,

$\boldsymbol{\Sigma}$ Conv: Total de postulantes, Cexp: Calificación de Expedientes, Eprelim: Entrevista preliminar.

\section{El Programa de Selección de personal}

La Selección de personal es una de las etapas claves y decisivas en el Proceso de Captación de recursos humanos, en razón a que sus resultados deberán exitosamente satisfacer las necesidades del puesto de trabajo, y de los cuales la organización exige una oportuna y eficaz atención.

Ello exige al profesional responsable una ejecución con un alto nivel de experiencia profesional; así como, un conocimiento del planeamiento eficiente para la ejecución de cada uno de sus procesos de trabajo.

La amplitud o profundidad de los procesos selectivos varía de acuerdo a las políticas de la organización, y para garantizar un trabajo de esta trascendencia se considera básicamente en su ejecución la: 
1. La Evaluación Técnica, orientada a la determinación del nivel de conocimiento teórico práctico que posee el postulante sobre el puesto de trabajo.

Su ejecución puede efectuarse por modalidades de: exámenes orales y/o escritos, de tipo laboratorio para exponer la solución de problemas de un proceso de trabajo propuesto que exige el puesto, la evaluación en el mismo puestode trabajo sobre procesos efectivos en tiempo real, etc.

2. La Evaluación Psicológica, está orientada al conocimiento de los componentes siguientes: el nivel intelectual, en relación al nivel de responsabilidad de puesto de trabajo (razonamiento general, razonamiento mecánico, etc.), las aptitudes (habilidades, destrezas, capacidad de aprendizaje, creatividad, etc.), las actitudes (tipos de organización, ideología, creencias, etc), las tendencias en los principales factores de la personalidad (contacto social y estabilidad emocional basicamente); asimismo, de acuerdo a la exigencia del nivel de responsabilidad del puesto de trabajo se evaluarán otros factores de la personalidad.

La aplicación del tipo de pruebas psicológicas, sean factorialistas, proyectivas, etc., es de responsabilidad del profesional designado, según su criterio y/o experiencia en el campo de la selección y evaluación de recursos humanos.

3. Las Entrevistas, herramientas valiosas en toda selección de personal, que a través de la observación y comunicación, permiten consistenciar y acopiar mayor información de los postulantes, motivo por el cual se resalta la utilidad de su aplicación en todo Programa de Selección de personal, precisando que no son un componente aislado, ellas se ejecutan en cada uno de los procesos del Programa.

Se desarrollan principalmente los siguientes tipos de entrevistas:

- La «Primera Entrevista» o entrevista técnica, que puede aplicarse antes o después de la evaluación técnica, recomendándose que esté a cargo del supervisor o jefe de área usuaria de la organización, donde va a laborar el postulante a seleccionar.

- La «Entrevista Psicológica» a cargo del profesional de la evaluación psicológica.

- La «Entrevista de Selección» o entrevista final, es la herramienta clave en la toma de decisión para elegir al candidato potencial que necesita la organización.

Para ejecutar la entrevista de selección deberá contarse con la información de los resultados de las evaluaciones ejecutadas.

Se recomienda que la elección del candidato sea mediante la presentación de ternas para cada tipo de puesto de trabajo, y que la decisión final de la escogencia sea por la opinión del jefe del área donde va a laborar el candidato ganador.

4. La Evaluación Médica se orienta al conocimiento del estado de salud del postulante en relación a su capacidad de respuesta para las exigencias de las labores del puesto de trabajo. Su ejecución o no dependerá de la política de la organización y de realizarse puede efectuarse antes o después de la evaluación técnica o de la evaluación psicológica. 


\section{El Programa de Inducción de personal}

Al programa de Inducción de personal también se le denomina como admisión, acogida e ingreso, es la actividad que cierra el proceso de Captación de recursos humanos.

La finalidad es la Inducción-Orientación del nuevo personal, entendiéndose como inducción al inicio de la «socialización organizacional» con el propósito de dar una visión global de la organización, sus proyectos, dar calidez a su ingreso, brindar una atmósfera laboral saludable, incluyendo los valores.

Por su parte la orientación tiene como finalidad el brindar información al nuevo personal de su posición laboral dentro de la organización, facilitando su rápida adaptación al ambiente de trabajo, de propiciarle el interés por su nuevo trabajo e inculcarle el sentimiento de seguridad y que su integración a la organización sea bajo una filosofía de calidad y excelencia laboral.

\section{EL PROCESO DE HABILITACIÓN DE RECURSOS HUMANOS}

El proceso de Habilitación en la gestión de recursos humanos es de suma importancia para la organización, debido a que tiene que desarrollar las funciones de planificar, organizar, ejecutar y controlar las acciones de sus Programas de Formación y Capacitación de personal, así como adecuarse a las situaciones que demanda la política de personal que puede considerarlo como parte selectiva o continuación eliminatoria para el nuevo personal.

La Formación y la Capacitación son generalmente programas de naturaleza contínua o permanente, como también cíclicos o eventuales cuando se les considera como parte del proceso selectivo de nuevo personal.

Esta distinción es vital para efectos de la estructuración del programa (objetivos, nivel de contenidos y de participante, duración) relacionados a las exigencias técnicas e individuales del puesto de trabajo.

\section{El Programa de Formación de Personal}

La Formación de personal es un programa importante en el Proceso de Habilitación de recursos humanos, cuyas acciones ejecutan contenidos que exige el puesto de trabajo de acuerdo a los niveles de responsabilidad de las funciones o labores a cumplir, que guardarán una correspondencia con los niveles de estudio que posee el trabajador o candidato, y /o a la política de personal con respecto a los planes de carrera en la organización.

De esta manera la administración de sus acciones se desarrollará de menor a mayor exigencia de aprendizaje determinado por el puesto de trabajo, cuya meta es la de tecnificar a los trabajadores (o candidatos), de esta forma serán diseñados los eventos de:

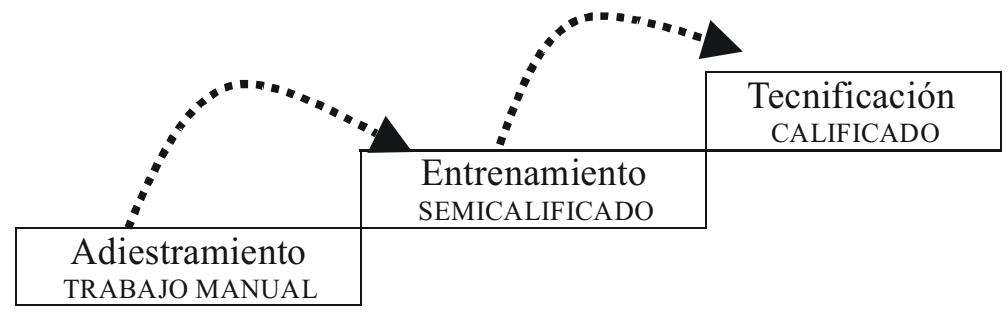


El objetivo-usuario es lograr la habilitación en los puestos de trabajo manual o «no calificados», «semicalificados» $\mathrm{y}$ «calificados».

\section{El Programa de Capacitación de Personal}

La Capacitación de personal es un programa importante en el Proceso de Habilitación de recursos humanos, cuyas acciones debidamente planificadas brindarán contenidos que exige el puesto de trabajo según los niveles de responsabilidad de las funciones o labores que cumplen y /o a la política de personal con respecto a los planes de carrera de la organización. La administración diseñará las acciones para cada tipo de capacitación (Actualización, Especialización, Perfeccionamiento), y por la exigencia de aprendizaje, los trabajadores o candidatos deberán poseer como mínimo el nivel de estudios superiores (tecnológico o universitario), para tener acceso a estos eventos, y/o de acuerdo a las necesidades de la organización determinados por la situación-problema del nivel de responsabilidad del puesto de trabajo.

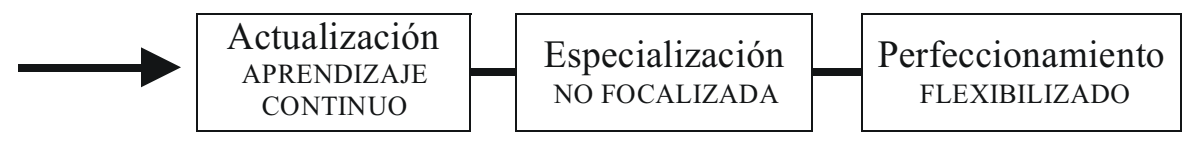

\section{EL PROCESO DE CALIFICACIÓN DE RECURSOS HUMANOS}

El Proceso de Calificación en la gestión de recursos humanos tiene relevante importancia debido a la información sobre la capacidad cuantitativa y cualitativa del personal, y que la obtiene mediante la aplicación de pruebas diseñadas para el logro de objetivos específicos, y/o que respondan a las situaciones que demanda la política de personal de la organización.

El Proceso de Calificación se instrumentaliza a través del Programa de Evaluación de personal.

\section{Los Programas de Evaluación de Personal}

Para atender los acelerados cambios que impone la globalización, la competitividad y las exigencias de los usuarios del mercado interno y externo, las organizaciones están aperturando variantes en el tradicionalmente conocido Programa de Evaluación de personal, que hasta el momento se aplican básicamente en tres tipos de pruebas de evaluación exploratoria referidas a:

- $\quad$ Evaluación del Desempeño del trabajador.

(Rendimiento)

- Evaluación de los Méritos del trabajador. (Innovación, creatividad)

- Evaluación de la Actuación del trabajador. $\quad$ (actitudes)

\section{Evaluación del Desempeño}

Tiene como objetivos principales el conocimiento del nivel de cumplimiento de las funciones, labores o tareas del trabajador en relación con los estándares asignados para el puesto de trabajo (rendimiento). 
De acuerdo a la política de la organización la Evaluación del Desempeño forma parte de la fase exploratoria de la evaluación de sus principales factores de rendimiento en cantidad y calidad, entre otros, y que a su vez sirve de marco referencial para la aplicación de la política de los planes de carrera de los trabajadores para efectos de promoción.

Existen software informáticos que procesan Programas de Evaluación del desempeño o rendimiento del trabajador, que permiten determinar factores en base a la agrupación de ítemes que guardan relación con los objetivos de la evaluación por rendimiento, y asignar el peso de cada factor de acuerdo a las prioridades de las políticas de evaluación del rendimiento y de los objetivos de perfiles por desempeño.

\section{Evaluación de Méritos}

Tiene como objetivo conocer las iniciativas que el trabajador ha desarrollado y que ha significado, en la mejora de los procesos de trabajo o de productos, es decir, aportes distintos al rendimiento en el puesto de trabajo.

La Evaluación de Méritos tiene varios propósitos:

- «Motivacional», que propicia una cultura organizacional de creatividad entre los trabajadores;

- «Incentivadora» que promueva una cultura integrativa y participativa de esfuerzo compartido para el beneficio común entre los trabajadores;

- «Exploratoria», que posibilita comportamientos orientados a la implementación o al mantenimiento de una cultura organizacional axiológica.

Según la política de la organización, el aspecto compensatorio por los aportes (creatividad, innovaciones, mejoramiento de procesos o procedimientos, etc.) que brindan los trabajadores, puede ser material y/o económico, recomendándose que los incentivos sean extensivos a los trabajadores del área de trabajo.

La Evaluación de Méritos refuerza la creatividad y la Evaluación de Desempeño o Rendimiento refuerza la productividad.

\section{Evaluación de la Actuación}

Tiene como objetivo conocer el nivel actitudinal de los trabajadores hacia la organización.

El diseño de Evaluación de la Actuación toma en cuenta objetivos específicos de la política de la organización relacionados a su crecimiento o desarrollo.

La finalidad es conocer y seleccionar a colaboradores de confianza que lleven a cabo la ejecución de la política de expansión y competitividad de la organización. Es la búsqueda interna de aliados estratégicos.

La Evaluación de la Actuación tiene las características siguientes:

- Evaluativa exploratoria sobre los niveles de identificación e internalización del trabajador con la cultura y política de la organización; 
- Selectiva para detectar y proponer personal como aliados estratégicos de la organización para garantizar las acciones o campañas de crecimiento y desarrollo de la organización.

\section{EL PROCESO DE POTENCIACIÓN DE RECURSOS HUMANOS}

El Proceso de Potenciación en la gestión de recursos humanos es de significativa importancia para la organización, debido a que se orienta a descubrir, estimular y poner en práctica las cualidades y potencialidades del personal que tiene la responsabilidad de conducir grupos humanos debidamente organizados (supervisores, jefes, gerentes, ejecutivos, directivos).

En esta perspectiva, en el Proceso de Potenciación se diseñan y ejecutan Programas de Desarrollo de personal, cuyos eventos deberán brindar nuevas técnicas, enfoques, herramientas e instrumentos que faciliten la obtención de logros exitosos en la gestión.

Hay organizaciones que consideran en su política de desarrollo empresarial / institucional el programa de Formación de Cuadros, que permite a los trabajadores que, no ejerciendo una responsabilidad de gestión, tienen antecedentes sobresalientes tanto en sus evaluaciones de rendimiento-desempeño, de actuación, de méritos, en eventos de formación y capacitación, y calificativos de ingreso a la organización, puedan asistir a los Programas de Desarrollo de Personal en calidad de participantes libres.

A este grupo de trabajadores la organización los considera como candidatos potenciales para acceder al reemplazo de cargos de función supervisora, jefatural, gerencial o directiva.

\section{TEMÁTICA DE REFLEXIÓN SOBRE EL FACTOR HUMANO EN LAS ORGANIZACIONES}

Es necesario resaltar, que actualmente las organizaciones vienen desarrollando procesos en la administración de recursos humanos que requieren del componente psicológico en el manejo de sus procesos o procedimientos para la certificación de la calidad y/o acreditación, para la aplicación de los nuevos esquemas en la negociación laboral, en la respondabilidad institucional y en su responsabilidad social como organización.

Los efectos de la globalización como consecuencia del desarrollo de la ciencia, contradictoriamente nos alejan de la «realidad», entendida ella como el espacio vital en el cual los seres humanos deben convivir mejor con una amplia y libre socialización, sin discriminaciones ni exclusiones, y que penosamente, vemos más distante esta «realidad» en la medida que aceledaramente avanza la tecnología.

El pragmatismo pretende ayudar convenciendo a que todo ello deberá asumirse como retos y desafíos, (cambios acelerados, competitividad, comunicación satelital, países sin fronteras, crecimiento económico sin desarrollo social, etc.,) y sin notarlo ingresamos a la vorágine de la tecnocracia sin rostro humano.

Las organizaciones vienen a ser el contexto social laboral de los seres humanos y el soporte productivo de la sociedad, y en ellas, no obstante la presencia del avance voraz de la tecnología no le garantiza su existencia, solamente el factor humano es el que decide su supervivencia, su desaparición ó su éxito. 
Ante esta realidad de inicio del siglo XXI se plantean nuevos escenarios para las organizaciones, que siendo la organización un sistema social proyectarla hacia un sistema inteligente, con más ciencia, más tecnología; no obstante este panorama de cercanía apocalíptica, se tratará de no alejarnos de la verdad, de la justicia social, de la sociedad libre, de la concertación, de los principios y los valores humanos. [8,9]

\section{REFERENCIAS BIBLIOGRÁFICAS}

1. Koontz, H / Weihrich, H. Administración: Una perspectiva global. 10. ${ }^{\mathrm{a}}$ ed. Editorial Mc Graw Hill.

2. Bohlander, Snell y Skerman (2001). Administración de recursos humanos. $12 .^{\mathrm{a}}$ ed. International Thomson Editores.

3. Perea R, J L. (2006). El planeamiento estratégico: En la perspectiva psicológica como producto de los procesos cognoscitivos para la dinámica humana y organizacional. Edición: Psicología Tópicos de Actualidad de la Escuela AcadémicoProfesional de la Facultad de Psicología de la UNMSM.

4. Senge M., P. (1999). La danza del cambio: Una herramienta para la quinta disciplina. Edit. Norma.

5. Perea R, J L. (2005). «Sistematización en una reforma curricular». En: Revista de Investigación en Psicología. Facultad de Psicología de la UNMSM.

6. Perea R. J L. (2000). «La psicología en los procesos de gestión de recursos humanos». Separata Curso Psicología Organizacional. Facultad de Psicología de la UNMSM.

7. Perea R, J L. (1994). El reclutamiento y selección de personal: Enfoque sistémico en el proceso de captación de los recursos humanos (Monografía). Facultad de Psicología de la UNMSM.

8. García Pérez, Alan (2004). Modernidad y política en el siglo XXI: Globalización con justicia social. Editorial Matices.

9. Vargas Llosa, Alvaro (2004). Rumbo a la libertad: Por qué la izquierda y el neoliberalismo fracasan en América Latina. Editorial Planeta.

\section{BIBLIOGRAFÍA DE CONSULTA}

1. Blanchard, K. Administración por valores. Editorial Norma.

2. Blanchard, K. Empowerment. Editorial Norma.

3. Beckhard, R. Desarrollo organizacional: Estrategias y modelos. Ed. Fondo Educativo

4. Bennis, Warren G. Desarrollo organizacional: Naturaleza, orígenes y perspectivas. Ed. FEI.

5. Boxwell Jr., Robert. Management Siglo XXI: Benchmarking. Ed. McGraw-Hill.

6. Berry, Thomas H. Management Siglo XXI: Calidad Total. Ed. McGraw-Hill. 
7. Best, L. Teoría de los valores y ética. Lima, Ediciones Univ. Garcilaso de la Vega.

8. Chiavenato, I. Administración de recursos humanos. Ed. Mc Graw Hill.

9. Chruden y Sherman. Administración de personal. Ed. Continental.

10. Davis y Newstrom. Comportamiento humano: Comportamiento organizacional. Ed. Mc Graw Hill.

11. Drucker, P. La gerencia en la sociedad futura. Editorial Norma.

12. Fleitman, J. Evaluación integral: Manual para el diagnóstico y solución de problemas de productividad, calidad y competitividad. Ed. Mc Graw Hill.

13. Gil' Adí Daniel. Inteligencia emocional en práctica. Manual para el éxito personal y organizacional. Ed. Mc Graw Hill

14. Hill, CH. y Jones, G. Administración estratégica: Un enfoque integrado. Ed. Mc Graw Hill.

15. Margulies, N. y Raia A. Desarrollo organizacional: Valores, procesos y tecnología.Ed. Diana.

16. Mc Donald, J. y Piggot J. Cambio de paradigmas empresariales. Ed. Panorama.

17. Mc Gregor, Douglas. El aspecto humano de las empresas. Ed. Diana.

18. Morris, D. y Brandon, J. Management siglo XXI: Reingeniería. Ed. Mc Graw Hill.

19. Nardone G. y Watzlawick . El arte del cambio. Ed. Herder.

20. Nash, M. Productividad de los RR. HH.Cómo incrementarla. Ed. Norma.

21. Perea R, J L. Cultura organizacional. Separata Psicología Organizacional II.

22. Peters, Walter. En busca de la excelencia. Ed. Norma.

23. Pezo, A. Gestión estratégica del capital humano. Ediciones del Programa Forte-PE.

24. Robbins, S. Comportamiento organizacional: Fundamentos. Ed. Prentice Hall.

25. Senge M., P. La quinta disciplina: El arte y práctica de la organización abierta al aprendizaje. Ed. Granica.

26. Senge M., P. La quinta disciplina en la práctica: Estrategias y herramientas para construir la organización abierta al Aprendizaje. Ed. Granica.

27. Soto, E. Comportamiento organizacional: Impacto de las emociones. International Thomson Editorial. 